\title{
Síndromes Hipertensivas Gestacional e o manejo da Enfermagem no âmbito da
}

\section{Atenção Primaria}

Gestational Hypertensive Syndromes and Nursing management in the framework of Primary Care Síndromes Hipertensivos Gestacionales y manejo de Enfermería en el marco de la Atención

\section{Primaria}

Rita Maria Ramalho da Silva

ORCID: https://orcid.org/0000-0002-4256-3210 Faculdade de Tecnologia e Educação Superior Profissional, Brasi

E-mail: rita.ramalho1@ hotmail.com

Samuel Lopes dos Santos

ORCID: https://orcid.org/0000-0003-3375-9171

Universidade Federal do Piauí, Brasil

E-mail: samuellopes121314@gmail.com

Thyago de Oliveira Afonso

ORCID: https://orcid.org/0000-0001-7616-9011

Universidade Federal de Pernambuco, Brasil

E-mail: thyago.oafonso@gmail.com

Kellyane Folha Gois Moreira

ORCID: https://orcid.org/0000-0001-6451-5925

Universidade Federal do Piauí, Brasil

E-mail: kelly_folha@hotmail.com

Sara da Silva Siqueira Fonseca

ORCID: https://orcid.org/0000-0002-2209-5501

Universidade Federal do Piauí, Brasil

E-mail: ss.siqueira@hotmail.com

Patrícia Pereira dos Santos Silva

ORCID: https://orcid.org/0000-0002-9510-4704

Faculdade de Tecnologia e Educação Superior Profissional, Brasil

E-mail: patriciasantos474@yahoo.com

Pedro Lucas Alves Ferreira

ORCID: https://orcid.org/0000-0002-1975-5054

Universidade Federal do Piauí, Brasil

E-mail: p.lucasnutricionista@gmail.com

Higor Kardek Firmino da Silva

ORCID: https://orcid.org/0000-0002-3179-0557

Universidade Federal do Piauí, Brasil

E-mail: higorkardek@gmail.com

Cristian de Sousa Sabino

ORCID: https://orcid.org/0000-0002-4782-9495

Universidade Federal do Piauí, Brasil.

E-mail: csabino.sousa@gmail.com

Jessica Sabrina Rodrigues

ORCID: https://orcid.org/0000-0003-3280-7733

Universidade Paulista, Brasi

E-mail: j.srodrigues@live.com

Rhelryson Carlos Medeiros Costa

ORCID: https://orcid.org/0000-0003-2640-2529

Centro Universitário UNIFACID, Brasi

E-mail: rhmedeiros29@gmail.com

Héverson Batista Ferreira

ORCID: https://orcid.org/0000-0002-0697-6982 Universidade Potiguar, Brasil

E-mail: eversonbatst@gmail.com

Winícius de Carvalho Alves

ORCID: https://orcid.org/0000-0003-3118-5696

Universidade Federal de Pelotas, Brasil

E-mail: winiciusdecarvalho@hotmail.com

Yorran Ferreira Brito

ORCID: https://orcid.org/0000-0003-1475-3049

Centro Universitário UNIFAPI, Brasil

E-mail: Yorrandu13@hotmail.com 


\begin{abstract}
Resumo
Os mecanismos patogênicos que causam as crises hipertensivas ainda são desconhecidos, porém, existem causas que determinam e facilitam o seu aparecimento como estresse, condições nutricionais precárias, raça negra, início da vida reprodutiva, nível socioeconômico, obesidade, tabagismo, diabetes mellitus, antecedentes familiares e hipertensão arterial. O objetivo do estudo foi analisar as evidências disponíveis na literatura sobre as condutas dos enfermeiros no cuidado às gestantes com síndrome hipertensiva específica gestacional (SHEG). Trata-se de um estudo de Revisão Integrativa da Literatura com análise descritiva e qualitativa, realizado nas bases de dados eletrônica da LILACS e nas bibliotecas eletrônicas SciELO e BVS. Foram incluídos estudos primários que contemplaram a temática, produzidos em língua inglesa, portuguesa e espanhola no período de janeiro de 2015 a julho de 2021. E excluídos, estudos incompletos, duplicados e os de domínio privado, impossibilitando a leitura na integra do estudo. Evidenciou-se que as ações executadas pelos enfermeiros são atividades de cunho preventivo como o acompanhamento gestacional precoce, busca de soluções às demandas por meio do referenciamento das RAS, identificação de níveis elevados de pressão arterial nas consultas, classificação do pré-natal dentre outras. Conclui-se que as SHEG são fatores agravantes para a gestante, podendo evoluir para complicações severas, como fim da gestação, indução ao parto cirúrgico de urgência. Ressalta-se que o enfermeiro é um profissional indispensável e essencial para oferta do cuidado integral para a saúde das gestantes e manejo as síndromes hipertensivas.
\end{abstract}

Palavras-chave: Crise hipertensiva; Gestante; Enfermagem.

\begin{abstract}
The pathogenic mechanisms that cause hypertensive crises are still unknown, however, there are causes that determine and facilitate their onset such as stress, poor nutritional conditions, black race, early reproductive life, socioeconomic status, obesity, smoking, diabetes mellitus, family history and high blood pressure. The aim of the study was to analyze the evidence available in the literature on the behavior of nurses in caring for pregnant women with gestational specific hypertensive syndrome (SHEG). This is an Integrative Literature Review study with descriptive and qualitative analysis, carried out in LILACS electronic databases and in SciELO and BVS electronic libraries. Primary studies that addressed the theme, produced in English, Portuguese and Spanish from January 2015 to July 2021 were included. Incomplete studies, duplicates and those in the private domain were excluded, making it impossible to read the entire study. It was evident that the actions performed by nurses are preventive activities such as early gestational follow-up, search for solutions to demands through the referral of RAS, identification of high levels of blood pressure in consultations, classification of prenatal care, among others. It is concluded that SHEG are aggravating factors for pregnant women, and may evolve into severe complications, such as the end of pregnancy, induction of urgent surgical delivery. It is noteworthy that the nurse is an indispensable and essential professional to offer comprehensive care for the health of pregnant women and management of hypertensive syndromes.
\end{abstract}

Keywords: Hypertensive crisis; Pregnant; Nursing.

\title{
Resumen
}

Aún se desconocen los mecanismos patogénicos que provocan las crisis hipertensivas, sin embargo, existen causas que determinan y facilitan su aparición como el estrés, malas condiciones nutricionales, raza negra, vida reproductiva temprana, nivel socioeconómico, obesidad, tabaquismo, diabetes mellitus, antecedentes familiares y Alta presión sanguínea. El objetivo del estudio fue analizar la evidencia disponible en la literatura sobre el comportamiento del enfermero en el cuidado de la gestante con síndrome hipertensivo específico gestacional (SHEG). Se trata de un estudio de Revisión Integrativa de Literatura con análisis descriptivo y cualitativo, realizado en las bases de datos electrónicas LILACS y en las bibliotecas electrónicas SciELO y BVS. Se incluyeron los estudios primarios que abordaron el tema, producidos en inglés, portugués y español desde enero de 2015 hasta julio de 2021. Se excluyeron los estudios incompletos, duplicados y del dominio privado, imposibilitando la lectura del estudio completo. Se evidenció que las acciones que realizan los enfermeros son actividades preventivas como seguimiento gestacional precoz, búsqueda de soluciones a demandas a través de la derivación de RAS, identificación de niveles altos de presión arterial en las consultas, clasificación de la atención prenatal, entre otras. Se concluye que los SHEG son factores agravantes para las mujeres embarazadas y pueden evolucionar a complicaciones graves, como el final del embarazo, la inducción de un parto quirúrgico urgente. Es de destacar que la enfermera es un profesional indispensable e imprescindible para ofrecer una atención integral a la salud de la gestante y manejo de los síndromes hipertensivos.

Palabras clave: Crisis hipertensiva; Embarazada; Enfermería.

\section{Introdução}

As Síndromes Hipertensivas Especificas durante a Gestação (SHEG) estão presentes em 10 a $22 \%$ das mulheres grávidas, contudo, estudos mostram que quando não são aplicadas intervenções adequadas para tratamento do quadro, estas 
evoluem podendo ocasionar sérios problemas para a mulher e o feto, estimando-se que $90 \%$ das internações em UTIs obstétricas são relacionados pelo agravamento das SHEG (Coelho et al., 2019).

Vale ressaltar que, segundo a Organização Mundial da Saúde (OMS), as complicações hipertensivas encontram-se como a segunda causa de mortalidades maternas/fetais, junto às complicações hemorrágicas, estas são responsáveis por 14\% das mortes maternas no mundo, chegando a cerca de $22 \%$ na américa latina (Silva et al., 2020). No Brasil, em 2016, as complicações hipertensivas foram responsáveis por mais de 341 causa morte maternas (Brito et al., 2015).

Os mecanismos patogênicos que causam as crises hipertensivas ainda são desconhecidos, porém, existem causas que determinam ou facilitam o seu aparecimento, como estresse, condições nutricionais precárias, raça negra, início da vida reprodutiva, nível socioeconômico, obesidade, tabagismo, diabetes mellitus, antecedentes familiares e hipertensão arterial (Aguiar, 2014).

Dentre os principais problemas hipertensivos na gestação, estão: a hipertensão gestacional; pré-eclâmpsia (PE); eclampsia superposta (EC) e a síndrome de HELLP (Queiroz \& Marcel, 2018).

$\mathrm{Na}$ "hipertensão gestacional", há um aumento da Pressão arterial (PA) para níveis de >140 x 90 mmHg, tendo seu primeiro diagnostico na gestação, ausência de proteinúria, e um retorno aos níveis tensionais em média de 12 semanas após o parto; "pré-eclâmpsia", é tida como um aumento da PA, associado ao quadro de proteinúria, manifesta-se com sinais e sintomas: oliguria, cefaleia intensa, desconfortos cardíacos etc. Já na "eclampsia" observa-se níveis pressóricos elevados e sustentados, presença de proteinúria acompanhado de crises convulsivas podendo haver também restrição de crescimento intrauterino e/ou oligohidrâmnio e por fim, "a Síndrome de HELLP", caracteriza pelo quadro de plaquetopenia abrupta, alterações nas enzimas hepáticas e hemólises (Coelho et al., 2019).

O que essas síndromes tem em comum, é que necessitam de identificação precoce e um manejo intervencional apropriado e rápido. Assim, a atenção básica, responsável pela maioria dos acompanhamentos gestacionais de baixo e médio risco, é o local onde se espera os primeiros atendimentos a gestante, devido a sua proximidade com a gestante e está frequentemente em contato com esta, nas consultas de pré-natal (Pozza et al., 2016).

O enfermeiro, é um dos profissionais habilitados a realizarem o acompanhamento de pré-natal as gestantes, geralmente após a captação da gestante, ocorrem o encaminhamento para a primeira consulta, realizada pelo enfermeiro (Aguiar, 2014). Na avaliação, é o momento de identificar riscos potenciais ao desenvolvimento de complicações gestacional, traçar medidas de enfrentamento e orientar quanto o acometimento dessas possíveis complicações como, procurar de imediato a Unidade básica de saúde na ocorrência de problemas clínicos, corroborando assim para que este profissional seja o primeiro a identificar as síndromes hipertensivas gestacionais. (Pozza et al., 2016).

Diante desta problemática, o estudo teve como objetivo analisar as evidências disponíveis na literatura sobre as condutas dos enfermeiros no cuidado às gestantes com síndrome hipertensiva específica gestacional no âmbito da atenção básica a saúde.

\section{Metodologia}

Trata-se de uma Revisão Integrativa da Literatura, de caráter descritivo e qualitativa com seleções de informações a partir da revisão bibliográfica, método ao qual analisa-se um tema compreendido em pesquisas relevantes anteriores, possibilitando a análise do tema abordado e objetivando-se a compreensão deste assunto, em que há o favorecimento, caracterização e divulgação das informações produzidas (Fernandes et al., 2016).

Assim, este estudo seguiu a seguintes etapas, como descrita e evidenciadas no estudo de revisão sistemática: 1) levantamento do tema e problemática; 2) busca e seleção do material base para construção do estudo; 3) tratamento sistêmico 
dos dados e análises crítica dos dados; 4) formulação dos resultados e discussão do estudo; 5) conclusão pautada na fidedignidade e 6) apresentação da síntese final (Mendes; Silveira \& Galvão, 2008).

Para nortear a busca pelos artigos, previamente se estabeleceu a seguinte questão norteadora: Quais as condutas dos enfermeiros no cuidado às gestantes com síndrome hipertensiva específica gestacional na atenção básica em face de resposta a questão norteadora, estabeleceu-se o crômio PICo, P (Mulheres grávidas - síndrome hipertensiva), I (Ações e estratégias da enfermagem - atenção básica) e Co (ações - pré-natal).

A coleta de dados foi realizada nos meses de maio a julho de 2021nas base de dados: Biblioteca Virtual de Saúde (BVS) e Biblioteca Eletrônica do Sientific Eletronic Library Online (SciELO) e na base de dados LILACS. Para a localização dos estudos relevantes, que respondessem à questão norteadora da pesquisa foram utilizados os seguintes descritores: "Crise hipertensiva” AND “Gestante” AND “Enfermagem” obtidos a partir dos Descritores em Ciências da Saúde (DeCS).

Foram incluídos estudos primários que contemplaram a temática, produzidos em língua inglesa, portuguesa e espanhola no período de janeiro de 2015 a julho de 2021. E excluídos, estudos incompletos, duplicados e os de domínio privado, impossibilitando a leitura na integra do estudo.

A busca inicial resultou num quantitativo de 102 artigos nas três bases de dados utilizadas, destes, 84 foram excluídos após a aplicação dos critérios de inclusão e exclusão restando um total de 18 artigos para leitura e analises inicial, após a segunda etapa, foram descartados um total de 10 artigos por se classificar como inadequados a este estudo, resultando um total de 08 artigos para amostragem final. A construção deste estudo, foi realizado um levantamento teórico conforme estabelecido nos métodos e materiais do estudo, após isso, obteve-se a amostragem final de oito artigos, classificados como adequados para este estudo. A Figura 1, ilustra detalhadamente como se deu essa seleção. 
Figura 1. Seleção amostral dos artigos usados no estudo.

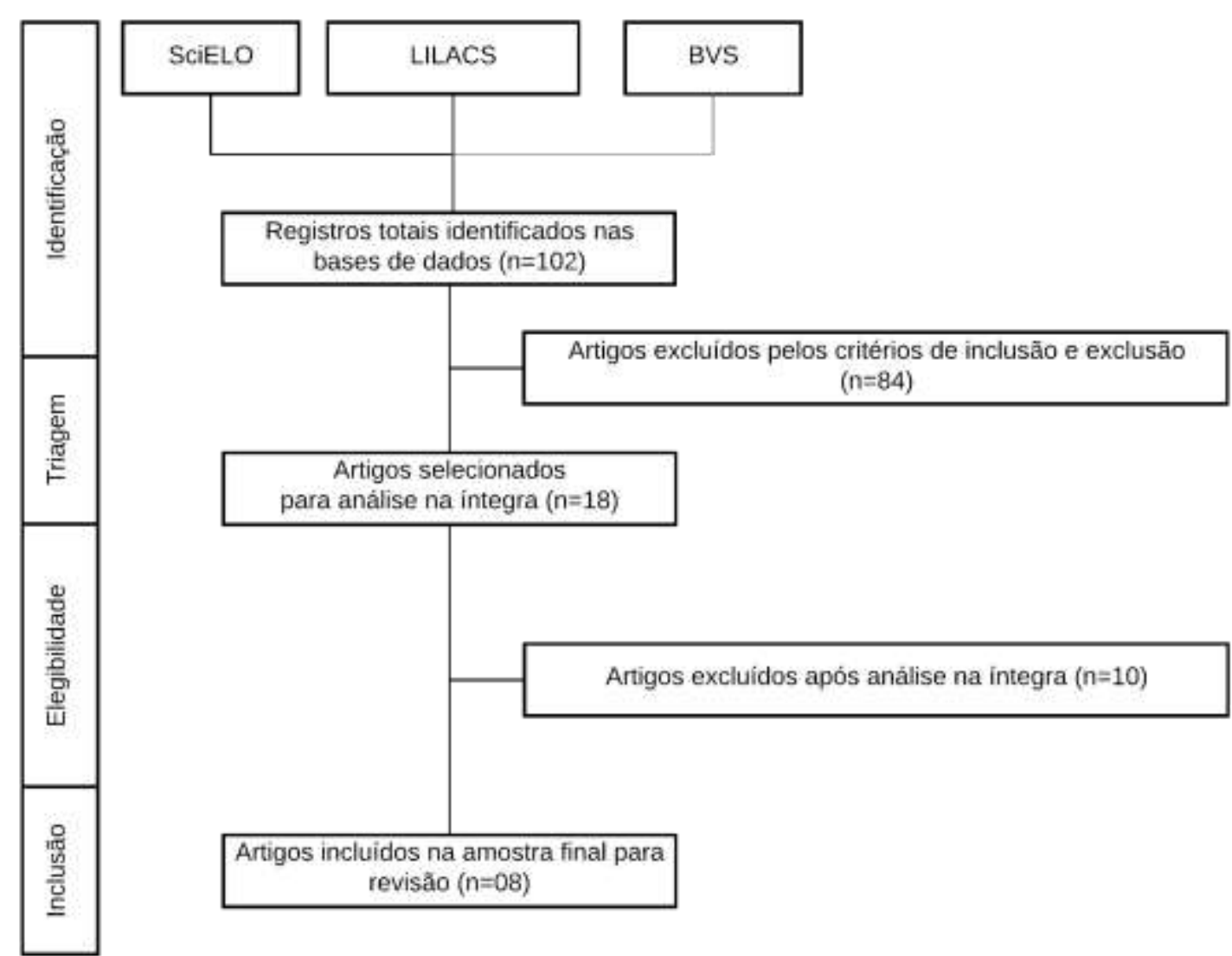

Fonte: Direta de estudo/autor.

Após a seleção dos artigos os dados foram organizados em um quadro contendo título, autores, ano, base de dados, e metodologia. E a discussão foi realizada por meio de Três categorias analíticas como Proposta por Bardin (2016), pré-análises, exploração do material e Tratamento dos resultados (inferência e apresentação).

\section{Resultados e Discussão}

Quadro 1. Descrição dos artigos quanto ao título, autor e ano, revista e metodologia. Teresina, Piauí, Brasil, 2021.

\begin{tabular}{|c|c|c|c|c|}
\hline $\mathrm{N}^{\mathrm{o}}$ & TITULO & AUTOR/ANO & BASE & METODOLOGIA \\
\hline 1 & cuidados de enfermagem a gestante com síndrome hipertensiva: revisão integrativa & $\begin{array}{l}\text { OLIVEIRA LEYLIANNE, } \\
\text { A. N; et al., } \mathbf{2 0 1 8}\end{array}$ & LILACS & Estudo Literário \\
\hline 2 & $\begin{array}{l}\text { Síndromes hipertensivas na gestação no Brasil: estudo a partir dos dados da pesquisa } \\
\text { "Nascer no Brasil: inquérito nacional sobre o parto e nascimento", 2011-2012 }\end{array}$ & $\begin{array}{l}\text { QUEIROZ, MARCEL REIS, } \\
\mathbf{2 0 1 8}\end{array}$ & SciELO & Estudo Transversal \\
\hline 3 & Prevalência das síndromes hipertensivas específicas da gestação & $\begin{array}{l}\text { BRITO KKG, Moura JRP, } \\
\text { Sousa MJ et al0., } 2015\end{array}$ & LILACS & $\begin{array}{l}\text { Estudo Descritivo } \\
\text { Retrospectivo }\end{array}$ \\
\hline 4 & $\begin{array}{l}\text { Construção e validação de escala de autoeficácia de gestantes na prevenção de } \\
\text { complicações das síndromes hipertensivas da gravidez }\end{array}$ & CHAVES, A. C.M, 2019. & LILACS & $\begin{array}{l}\text { Estudo Metodológico } \\
\text { Experimental }\end{array}$ \\
\hline 5 & $\begin{array}{l}\text { Ocorrência de complicações no período gestacional em mulheres com idade materna } \\
\text { avançada }\end{array}$ & $\begin{array}{l}\text { RIBEIRO, A. J. D.; et al., } \\
\mathbf{2 0 2 1}\end{array}$ & BVS & $\begin{array}{l}\text { Estudo Descritivo } \\
\text { Quantitativo }\end{array}$ \\
\hline 6 & Nursing's action against patients with specific hypertensive management syndrome & $\begin{array}{l}\text { MARTINS, A. A. C; et al., } \\
\mathbf{2 0 2 0}\end{array}$ & BVS & Estudo Experimental \\
\hline 7 & $\begin{array}{l}\text { Implementation of a standardized nurse initiated protocol to manage severe } \\
\text { hypertension in pregnancy. }\end{array}$ & MILLER, M. J. et al., 2020 & SciELO & $\begin{array}{l}\text { Estudo Longitudinal e } \\
\text { Descritivo }\end{array}$ \\
\hline 8 & $\begin{array}{l}\text { Perfil socioeconômico, demográfico e obstétrico de gestantes com Síndrome } \\
\text { Hipertensiva de uma maternidade pública }\end{array}$ & SILVA, J. L.M, et al., 2020 & LILACS & $\begin{array}{l}\text { Estudo Descritivo } \\
\text { Correlacional }\end{array}$ \\
\hline
\end{tabular}

Fonte: Autores. 
Na interpretação dos resultados, nota-se que o ano de 2020 foi destaque com maior publicação, sendo um total de três artigos usados, seguido de 2018 com dois artigos, e a principal base dados foi a LILACS, com quatro artigos, dentre o método, destacou-se o estudo do tipo descritivo com três artigos, seguido do estudo experimental com dois artigos.

\section{Fatores associados à Síndrome Hipertensiva Especifica Gestacional (SHEG)}

Segundo Brito e colaboradores (2021), diversos fatores estão associados ao maior risco de SHEG, como idade materna avançada, diabetes mellitus, obesidade, doença renal, colagenoses, história familiar de SHEG, gestação múltipla, doença trofoblástica gestacional, SHEG em gestação pregressa, entre outros, podem ser apontados como fatores predisponentes. Além disso, a maioria dos casos dessa doença, cerca de 75\%, ocorre em mulheres nulíparas. Nesses casos, a doença é mais observada em pacientes com hipertensão arterial pré-gravídica ou quando há troca de parceiro (Queiroz, 2018).

No entanto, independente da paridade, na gestação múltipla, a doença hipertensiva tende a ocorrer em idades gestacionais mais precoces quando comparadas a gestações únicas e são mais graves. Em relação ao nível socioeconômico, não há evidências conclusivas de que isso possa ter relação com a síndrome hipertensiva (Júnior et al., 2019).

Porém, a eclampsia e a síndrome HELLP são mais frequentes nas camadas socioeconômicas menos favorecidas. Apesar de não ser consenso, formas mais avançadas da doença hipertensiva pode decorrer do fato de que há falta de assistência pré-natal ou assistência médica precária nas classes menos favorecidas (Coelho, 2019; Gracia, 2015; Guimarães et al., 2014).

Um fator bem estabelecido é a maior presença de DHEG em extremos de idade, ou seja, abaixo dos 18 e acima dos 40 anos. Isso pode ser relacionado a nuliparidade em pacientes mais jovens e nas mulheres com idade avançada, a hipertensão arterial essencial, embora mais frequente, favorece a instalação da DHEG (Ribeiro et al., 2021).

Alguns estudos evidenciaram a predisposição hereditária à SHEG, por meio da participação de polimorfismos de alguns genes. Ademais, a obesidade é um grande fator contribuinte para as síndromes hipertensivas, sendo que quanto maior o índice de massa corporal (IMC), maior o risco de DHEG. Sabe-se que o mecanismo fisiopatológico está relacionado à intolerância à glicose, o mesmo motivo que o risco de DHEG em pacientes com diabetes pré-gestacional é de cerca de $20 \%$ (Ribeiro et al., 2021; Silva et al., 2020).

Vale lembrar que a SHEG está presente com maior frequência nos casos de doença trofoblástica gestacional e nessa condição, surge em idades gestacionais mais precoces. Ademais, tais condições podem ser identificadas previamente no prénatal e seguir protocolos bem estabelecidos para controle (Martins et al., 2020; Miller et al., 2020; Oliveira et al., 2018).

\section{Cuidados da enfermagem para um adequado manejo da gestante com Hipertensão.}

Alguns cuidados são necessários para um manejo adequado as gestantes com hipertensão, dentre eles, algumas ações e condutas a serem realizadas pelo enfermeiro que acompanha uma gestante com HAS na atenção primaria, como os listados no Quadro 2.

Quadro 2. Ações a serem tomadas pelo enfermeiro no manejo a Gestante com HAS.

o Classificação do risco gestacional;

o Realização de no mínimo seis consultas de pré-natal;

- Verificar atores de potencialização da HAS, como patologias de base e doenças intensificadoras;

- Verificar PA em todas as consultas, IMC, avaliação fetal completa, ausculta cardiopulmonar e buscar por edema;

o Controle nutricional

o Controle emocional e identificação de patologias psicológicas

o Orientar quanto aos sinais de agravamento e condutas imediatas a serem tomadas.

$\begin{array}{lll}\sim & \sim & \sim \\ \end{array}$

Fonte: Autores (2021) 


\section{Atribuições do enfermeiro na prevenção e promoção da saúde.}

Segundo Fassarela e colaboradores (2020), os cuidados prestados pela enfermagem a gestante portadora de Hipertensão com fatores agravantes são essenciais e indispensáveis, dentre eles destacam-se os eventos preventivos como Orientação quanto ao consumo de drogas e uso de álcool durante a gestação, fatores estressores, condições psicológicas e fatores nutricional. Corrobora com o estudo de Thuler (2018) que enfatiza os cuidados preventivos como ações de controle ao agravamento da Hipertensão em gestantes e primeira linha de cuidados contra as síndromes hipertensivas gestacional mais comprometedora.

É valido considerar, as síndromes psicológicas são um potencial agravante quando considerado para efeito de elevação dos níveis pressóricos, a ansiedade, isolamento social, depressão, sentimentos de angustia, não aceite da gravidez dentre outros, devem ser identificados nas primeiras consultas pelo enfermeiro e traçado medidas de enfrentamento direta a esses problemas, como solicitação de auxilio por outros profissionais, além de traçar um plano de cuidados a essa gestante, durante todo o processo gravídico (Thuler et al, 2018).

O enfermeiro deve incentivar a pratica de exercícios físicos leves como a caminhada diária com periodicidade não superior a $30 \mathrm{mim}$; práticas de alimentação saudável com vista a prevenir a obesidade e as doenças oportunista dessa patologia como: acidentes cardiovasculares; problemas respiratórios e do sistema nervoso central (Oliveira et al. 2018).

A aplicação do processo de enfermagem é essencial desde histórico, diagnostico, planejamento, implementação e avaliação de forma continua, interrelacionadas e recorrentes, sendo assim, capaz de aplicar medidas diretas de enfrentamento a hipertensão. Vale destacar que, a solicitação de exames de rotina para pré-natal bem como a prescrição de medicamentos pelo profissional de enfermagem é indispensável no processo de prevenção e manejo as SHEG (Silva et al., 2020).

Frente ao quadro estabelecido de elevação dos níveis pressóricos, sugere-se o uso de anti-hipertensivos como: labetolol, nifedipina e metildopa, além da realização de exame de proteinúria de $24 \mathrm{~h}$, ECG e creatina. Caso descartado hipóteses de pré-eclâmpsia ou eclampsia, reavaliar e orientar retorno dentro de 15 dias, caso haja indícios sugestivo de patologias agravantes, encaminhar ao centro de referência obstétrica para avaliação (Martins et al., 2020).

É importante ressaltar que, no âmbito da atenção básica, as ações e intervenções de enfermagem as SHEG estão associadas/ligadas a cunhos preventivos, identificação, intervenção simples, encaminhamento para média e alta complexidade quando necessário e contrarreferência (Ribeiro et al., 2021).

Por fim, o cuidado deve ser ofertado de maneira holística, integralizado e acima de tudo humanizado, buscando-se o estabelecimento de vinculo e confiança entre a gestante e o profissional, se pôr como um profissional capaz de desenvolver uma "ausculta qualificada" e ser resolutivo (Martins et al., 2020).

\section{Conclusão}

As síndromes hipertensivas constituem-se como um potencial fator de morbimortalidade, devendo ser identificadas o quanto antes, a fim de tratar e controlar seus impactos à mãe e ao seu bebê. Dentre os fatores que contribuem para esta condição, estão: idade superior aos 40 anos e inferior aos 17, condições precárias de nutrição e alimentação, os fatores intrínsecos e extrínsecos como cor da pele parda, fatores familiares, histórico pregresso de alguma síndrome hipertensiva, primigesta e dentre outras.

No que concerne as atribuições da enfermagem, há ênfase para ações diretamente ligadas a realização das consultas de pré-natal, solicitação de exames complementares e de rotina, prescrição de medicamentos, orientação quanto as boas práticas de saúde (exercícios físicos, alimentação e nutrição ), rastreamento das mulheres com HAS crônico, bem como dos fatores que possam contribuir com o processo de vulnerabilidade a colocação e/ou agravamento de uma SHEG, destacando que essas ações de enfermagem são indispensáveis ao cuidado da gestante níveis elevado da PA. 
Research, Society and Development, v. 10, n. 15, e369101522060, 2021

(CC BY 4.0) | ISSN 2525-3409 | DOI: http://dx.doi.org/10.33448/rsd-v10i15.22060

\section{Referências}

Aguiar, L. R. S. et al. (2014) Análise de estudos sobre as condutas de enfermagem no cuidado a gestante com doença hipertensiva. Revista Interdisciplinar. $7(1), 204-215$.

Brasil. (2012) Ministério da Saúde. Gestação de alto risco: Manual técnico, (5a ed.).

Coelho, F.F, et al., (2019) Emergência Hipertensiva Na Gestação: Síndrome Hellp Uma Revisão De Literatura. Revista Saúde e Desenvolvimento. 12(13), 159175 .

Fernandes, M. G. M., et al., (2016) Análise de teorias de enfermagem de Meleis: revisão integrativa. Revista Brasileira de Enfermagem. 69(1), $174-181$.

Gil, A. C. (2017) Como elaborar projetos de pesquisa. (6a ed.). Atlas.

Guimarães, J. P. et al. (2014) A prevalência de gestantes portadoras de SHEG que evoluíram para síndrome HELLP. Revista Brasileira de Educação e Saúde. 4(1).

Júnior, F. S. B. et al. (2019) Perfil antropométrico de gestantes internadas com diagnóstico de pré-eclâmpsia grave. Journal of Nursing and Health. 9(3).

Oliveira A. N, et al. (2018) Cuidados de enfermagem a gestante com síndrome hipertensiva: revisão integrativa. Brazilian Journal of Surgery and Clinical Research-BJSCR. 23(2), 159-164.

Pozza, L. V. et al., (2016) Crise hipertensiva gestacional. Acta méd. 5-5.

Silva, A. M. et al. (2020) O Enfermeiro Perante A Hipertensão estacional. Revista Iniciare. 2(1).

Queiroz (2018) Síndromes hipertensivas na gestação no Brasil: estudo a partir dos dados da pesquisa "Nascer no Brasil: inquérito nacional sobre o parto e nascimento", 2011-2012. USP. https://doi.org/10.11606/T.6.2018.tde-23042018-140322

Brito, K. K. G., Moura, J. R. P., \& Sousa, M. J., et al., (2015) Prevalência das síndromes hipertensivas específicas da gestação. J. res.: fundam. care. online (3):2717-2725.

Chaves, A. C. M, (2019) Construção e validação de escala de autoeficácia de gestantes na prevenção de complicações das síndromes hipertensivas da gravidez. UFPR.

Ribeiro, A. J. D., et al., (2021) Ocorrência de complicações no período gestacional em mulheres com idade materna avançada. Rev. baiana enferm , 35: e43083, https://pesquisa.bvsalud.org/portal/resource/pt/biblio-1279765

Silva, J. L.M, et al., (2020) Perfil socioeconômico, demográfico e obstétrico de gestantes com Síndrome Hipertensiva de uma maternidade pública. Rev. gaúch. enferm 41: e20190180, https//pesquisa.bvsalud.org/portal/resource/pt/biblio-1101681

Miller, M. J. et al., (2020) Implementation of a standardized nurse initiated protocol to manage severe hypertension in pregnancy. J Matern Fetal Neonatal Med, 33(6): 1008-1014.

Martins, A. A. C, et al., (2020) Nursing's action against patients with specific hypertensive management syndrome. Rev. Cient. da Esc. Estadual de Saúde Pública de Goiás "Candido Santiago".

Thuler, A. C. M. et al., (2018) Medidas preventivas das sindromes hipertensivas da gravidez na atenção primária. Rev. Enferm UFPE on line. 